\title{
Characteristics of gram-negative urinary tract infections caused by extended spectrum beta lactamases: pivmecillinam as a treatment option within South Dublin, Ireland
}

Fardod O'Kelly ${ }^{2 \dagger}$, Siobhan Kavanagh ${ }^{1 \dagger}$, Rustom Manecksha ${ }^{2}$, John Thornhill ${ }^{2}$ and Jérôme P. Fennell ${ }^{1 *}$

\begin{abstract}
Background: The prevalence of urinary tract infections (UTIs) caused by extended-spectrum $\beta$-lactamase (ESBL)producing Enterobacteriaceae is increasing and the therapeutic options are limited, especially in primary care. Recent indications have suggested pivmecillinam to be a suitable option. This pilot study aimed to assess the viability of pivmecillinam as a therapeutic option in a Dublin cohort of mixed community and healthcare origin.

Methods: A prospective measurement of mean and fractional inhibitory concentrations of antibiotic use in 95 patients diagnosed with UTI caused by ESBL-producing Enterobacteriaceae was carried out. $36 \%$ patients were from general practice, $40 \%$ were admitted to hospital within south Dublin, and $25 \%$ samples arose from nursing homes. EUCAST breakpoints were used to determine if an isolate was sensitive or resistant to antibiotic agents.

Results: Sixty-nine percent of patients $(N=66)$ with urinary ESBL isolates were female. The mean age of females was 66 years compared with a mean age of 74 years for males. Thirty-six percent of isolates originated from primary care, hospital inpatients (26\%), and nursing homes (24\%). The vast majority of ESBL isolates were E. coli ( $80 \%)$. The $\mathrm{E}$ tests for mecillinam and co-amoxiclav had concentration ranges from $0.16 \mathrm{mg} / \mathrm{L}$ up to $256 \mathrm{mg} / \mathrm{L}$. The mean inhibitory concentration (MIC) of mecillinam ranged from 0.25 to 256 mg/L, while co-amoxiclav MICs ranged from 6 to $256 \mathrm{mg} / \mathrm{L}$. The percentage of isolates resistant to mecillinam and co-amoxiclav was found to be 5.26 and $94.74 \%$ respectively.

Conclusions: This is the first study exploring the use of pivmecillinam in an Irish cohort and has demonstrated that its use in conjunction with or without co-amoxiclav is an appropriate and useful treatment for urinary tract infections caused by ESBL-producing organisms.
\end{abstract}

Keywords: Extended-spectrum beta lactamase, Pivmecillinam, Antibiotic resistance, Urinary tract infection, Mean inhibitory concentration, Escherichia coli

\footnotetext{
* Correspondence: jfennell@tcd.ie; jerome.fennell@amnch.ie

${ }^{\dagger}$ Equal contributors

${ }^{1}$ Department of Clinical Microbiology, AMNCH, Tallaght Hospital, Dublin 24,

Ireland

Full list of author information is available at the end of the article
} 


\section{Background}

Microorganisms constantly evolve resistance to antimicrobials, rendering current agents ineffective. This is compounded by the reality that there are few new antimicrobials in development. Extended-spectrum beta lactamase (ESBL) producing organisms are one of the resistance types of most concern. ESBLs were first recognized in the 1980s due to point mutations of Temoneira (TEM) and Sulphydryl Variable (SHV) broad-spectrum enzymes genes. A common causes of hospital-acquired infections especially in the intensive care unit, ESBLs also commonly carry resistance to other antimicrobials such as quinolones, cotrimoxazole and the aminoglycosides. This further limits treatment options. Laboratory diagnosis of ESBLs is complex and normally performed by screening and phenotypic tests [1].

Traditionally ESBL producing bacteria were associated with nosocomial infection, but they are now widespread in the community. In the UK, community ESBLs were mostly isolated during urinary infections the elderly, who had recent hospitalisation [2]. In contrast, an Irish study found that $42 \%$ of community isolates that were ESBL producing, were from individuals not in long term care or hospitalised in the previous year [3]. Risk factors for acquiring community associated ESBL infections include recurrent UTI, previous antibiotic usage, diabetes and prior instrumentation to urinary tract $[1,4]$.

ESBLs were first detected in Western Europe, where $\beta$ lactam antibiotics were first used, with prevalence varying between countries. In 2001, the Netherlands had a low percentage of ESBL producing Enterobacteriaceae with only $1 \%$ of $E$. coli and $K$. pneumoniae being ESBL positive. In contrast $40 \%$ of France's $K$. pneumoniae were ceftazidime resistant. ESBL producers are normally multiple drug resistant and have become an important mechanism of $\beta$-lactam resistance in community uropathogens [5]. Production of $\beta$-lactamase is the most common resistance mechanism of bacteria to $\beta$-lactam antibiotics [6]. E. coli resistance is mostly due to production of $\beta$-lactamases, which hydrolyze the beta-lactam ring of beta-lactam antibiotics such as penicillin. Resistance to ampicillin and amoxicillin is normally due to plasmid-coded $\beta$ lactamases the majority of which is the TEM type [7]. ESBLs have resistance to $\beta$-lactams, ampicillin, amoxicillin and third generation cephalosporins. The ESBL carrying plasmid often carries other resistance genes as well, e.g. resistance genes to quinolones and aminoglycosides. When this occurs usage of any of the classes of antimicrobials that the plasmid encodes resistance to will select for this multiple resistant isolate. The first ESBLs in $E$. coli were variants of the TEM or SHV $\beta$-lactamases, which could hydrolyze cefotaxime, ceftriaxone and ceftazidime, however the CTX-M-type bla gene has now become the commonest type. The CTX-M enzymes also appear to have a greater ability to spread and cause outbreaks $[2,8,9]$. CLSI recommendations state to only check for ESBLs in E. coli, Klebsiella pneumoniae, K. oxytoca and Proteus mirabilis, but all Gram-negative bacteria can be ESBL positive. In 2006, two E. coli ESBL isolates were associated with UTIs from two residents in an Irish nursing home. On review, five more patients in that nursing home were found to be ESBL positive [10]. This is a typical example of the transmission of antibiotic resistant bacteria in a vulnerable group of patients where long-term isolation is not viable and there is a need to control the spread of these organisms. A more recent study surveyed an Irish nursing home and found over $55 \%$ of residents were colonized by ESBL producers [11]. In Ireland in 2015, $10.6 \%$ of invasive E. coli and $13.3 \%$ of invasive $K$. pneumoniae isolates were found to be ESBL Positive, the highest annual percentage to date [12].

There is a lack of effective therapeutic options to combat ESBLs. Carbapenems, often regarded the antibiotic of choice, should be used when there are no other options available but their use inevitably leads to the emergence of carbapenem-resistant enterobacteriaciae (CRE). Fluoroquinolones, can be effective against ESBLs, but are not recommended for routine use due to resistance rates. Aminoglycosides, also effective, should not be used for monotherapy in serious infections, as they are bacteriostatic. Colistin should be used with caution, as it is a broad-spectrum agent. The potential nephrotoxicity of these agents is another concern in this setting and is another reason to limit their use. Tigecycline demonstrates good in vitro activity against ESBLs but the FDA has warned against its use due to the increased mortality in Tigecycline-treated patients, as well as its relative inefficacy in pneumonia and bacteraemia, as well as limited GU tract concentrations [13, 14]. Fosfomycin, an old broadspectrum antibiotic, has been re-evaluated for the treatment of UTIs due to multidrug resistant organisms. It is only licensed for lower uncomplicated UTIs and may develop resistance [15].

Pivmecillinam, a $\beta$-lactam antibiotic, the prodrug of mecillinam, is hydrolyzed to the active agent mecillinam [16]. Mecillinam must be administered parentally but oral pivmecillinam is available. Its mode of action is to bind to penicillin-binding protein 2 in Enterobacteriaceae and inhibit bacterial cell wall synthesis [17]. It has high activity against many Gram-negative bacteria such as E. coli, Klebsiella sp., Salmonella sp. and Enterobacter $s p$. and has limited activity against some Gram positives. The use of pivmecillinam for more than 20 years in Nordic countries confirms its efficiency and safety in treating UTIs. Its oral bioavailability makes it an attractive option without requiring hospital admission for intravenous treatment $[16,18]$. 
Antibiotic resistance is a concerning public health problem that increases morbidity and mortality. Novel drug development is time-consuming, but re-evaluating antibiotics already licensed is more time-effective [17]. Combination therapy is of interest as multi drug resistant microorganisms may require more than one antibiotic to treat successfully [19]. Unfortunately, there are limited oral antibiotics available for complicated UTIs caused by ESBL and AmpC producing bacteria [15].

This clinical study examines the antimicrobial susceptibility of $95 \mathrm{ESBL}$ producing isolates to pivmecillinam and co-amoxiclav in a tertiary referral center, to determine if our catchment population could benefit with combination treatment of ESBL urinary tract infection.

\section{Methods}

This study received approval from the hospital ethics committee and the research carried out was also in compliance with the Helsinki Declaration. Clinico-demographic data was collated on 95 patients that had tested positive for ESBL urinary infection at the department of Microbiology, Tallaght hospital (2012-2013). Isolates had previously been confirmed as ESBLs by MASTDISCS ID AmpC and ESBL inhibitors and MASTDISCS cefepime ESBL ID for the Vitek 2. E-tests were performed on ESBLs that were initially stored on beads in an $-18{ }^{\circ} \mathrm{C}$ freezer, and then plated on MacConkey agar and incubated at $37^{\circ} \mathrm{C}$ for $24 \mathrm{~h}$. All isolates were then subcultured onto nutrient agar slopes. One mecillinam and one co-amoxiclav E test were then each applied with forceps onto the plate. The elliptical zones of inhibition were read to determine the MIC of $E$. coli according to the manufacturer instructions. The MIC was read as the point where the ellipse intersected the $\mathrm{E}$ test strip. If the intersect was different on both sides the greater value was taken as the MIC.

A checkerboard method was also used to assess the mean inhibitory concentrations (MIC) of each positive ESBL sample by measuring the spectrophotometric absorbance of each well 16 to $24 \mathrm{~h}$ following inoculation using an ELX800 universal micro plate reader at a wavelength of $630 \mathrm{~nm}$. The percentage of growth was calculated on the basis of colour absorbance using (OD630 of wells that contained the drug/OD630 of the drug-free well). The MICs of the drugs alone and of in combination were determined as the lowest drug concentrations showing $<10 \%$ of the growth of an untreated control [20, 21].

For each combination of antibiotics the fractional inhibitory concentration was calculated which is a predictor of synergy [22]. The fractional inhibitory concentration (FIC) was used to evaluate the effectiveness of the combinations. The formulas used were FIC of drug A (mecillinam) = MIC drug A in combination/ MIC drug A alone; FIC of drug B (co-amoxiclav) = MIC drug $\mathrm{B}$ in combination/ MIC drug B alone and FIC index = FIC drug A + FIC drug B. Synergy was defined as FIC index of $\leq 0.5$, indifference of FIC as $>0.5$ but of $\leq 4$. Antagonism was defined as FIC index of $>4$. All experiments were performed in triplicate.

EUCAST (European Committee on Antimicrobial Susceptibility Testing) breakpoints for Enterobacteriaceae for both mecillinam and co-amoxiclav were given as $\leq 8 \mathrm{mg} / \mathrm{L}$ as sensitive and $\geq 8 \mathrm{mg} / \mathrm{L}$ as resistant. However the British Society of Antimicrobial Chemotherapy states that an MIC of $\geq 32 \mathrm{mg} / \mathrm{L}$ for co-amoxiclav is suitable for UTIs but not systemic infections, due to the activity of clavulanate alone. Mecillinam concentrations in urine after $400 \mathrm{mg}$ taken orally has been reported to be above $100 \mathrm{mg} / \mathrm{L}$ after 6-h. Co-amoxiclav has also been found to have levels above the MIC in human serum during the first $6 \mathrm{~h}$ [23]. Therefore the EUCAST breakpoints were used to determine if the isolate was sensitive or resistant to both antibiotic agents. The red line in Figs. 1 and 2 illustrate the EUCAST breakpoint of $8 \mathrm{mg} / \mathrm{L}$.

\section{Results}

Sixty-nine percent of patients $(N=66)$ with urinary ESBL isolates were female. The mean age of females was 66 years compared with a mean age of 74 years for males. Thirty-six percent of isolates originated from primary care, hospital inpatients (26\%), and nursing homes (24\%). The vast majority of ESBL isolates were $E$. coli (80\%) (Tables 1 and 2).

The E tests for mecillinam and co-amoxiclav had concentration ranges from $0.16 \mathrm{mg} / \mathrm{L}$ up to $256 \mathrm{mg} / \mathrm{L}$. The mean inhibitory concentration (MIC) of mecillinam ranged from 0.25 to $256 \mathrm{mg} / \mathrm{L}$, while co-amoxiclav MICs ranged from 6 to $256 \mathrm{mg} / \mathrm{L}$. The MICs of mecillinam and co-amoxiclav were recorded and the total counts of the MIC were calculated. Distribution charts were constructed from this data (Figs. 1 and 2).

The percentage of isolates resistant to mecillinam and co-amoxiclav was found to be $5.26 \%$ and $94.74 \%$ respectively [24]. Four of five isolates were $E$. coli and were resistant to co-amoxiclav. Isolate number 513 was a Klebsiella species. These isolates were tested using the checkerboard technique for synergy between co-amoxiclav and mecillinam. The MIC was reduced significantly in isolates 842 and 513 in comparison to the E tests where only one drug was used. In the checkerboard method isolate 842 was inhibited with $24 \mathrm{ug} / \mathrm{ml}$ of co-amoxiclav in combination with $12 \mathrm{ug} / \mathrm{ml}$ of mecillinam. In the $\mathrm{E}$ test isolate 842 was inhibited by $12 \mathrm{ug} / \mathrm{ml}$ alone but was not inhibited by coamoxiclav until a concentration of $48 \mathrm{mg} / \mathrm{L}$ was used. Isolate 513 was inhibited at $24 \mathrm{ug} / \mathrm{ml}$ of co-amoxiclav in combination with $64 \mathrm{ug} / \mathrm{ml}$ mecillinam in the 96 well plate. In the $\mathrm{E}$ test isolate 513 was not inhibited by co-amoxiclav 


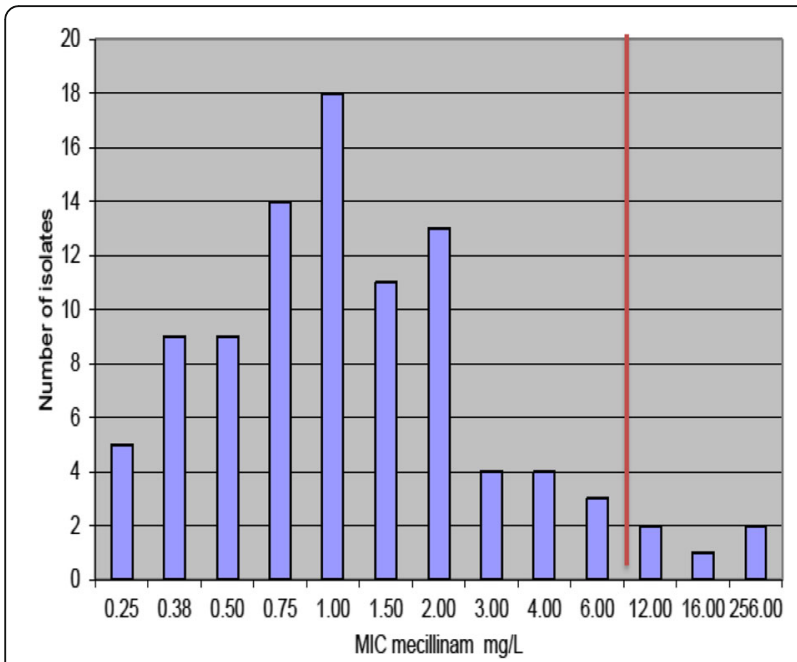

Fig. 1 Distribution of the mean inhibitory concentration (MIC) of 95 Extended spectrum beta-lactamase (ESBL) producing isolates. EUCAST break-point of $8 \mathrm{mg} / \mathrm{L}$ demonstrated by red vertical line

even at $256 \mathrm{mg} / \mathrm{L}$. Isolates 779 and 031 did not achieve levels of $10 \%$ of the growth of the control. Therefore the MIC for either or both drugs in combination was higher than $256 \mathrm{ug} / \mathrm{ml}$. In contrast the E test results showed isolate 779 was inhibited by co-amoxiclav at $128 \mathrm{mg} / \mathrm{L}$ but the results agreed with the checkerboard for mecillinam ( $>256 \mathrm{mg} / \mathrm{L}$ ). The E test showed that 031 was inhibited by $12 \mathrm{mg} / \mathrm{L}$ of mecillinam but was not inhibited by coamoxiclav even at $256 \mathrm{mg} / \mathrm{L}$. The FIC is correct in that no positive difference occurred when the two antibiotics were used in combination. Isolate 349 was inhibited by $128 \mathrm{mg} / \mathrm{L}$ of mecillinam alone and also in combination with co-amoxiclav (between 6-256 ug/ml) in the 96 well

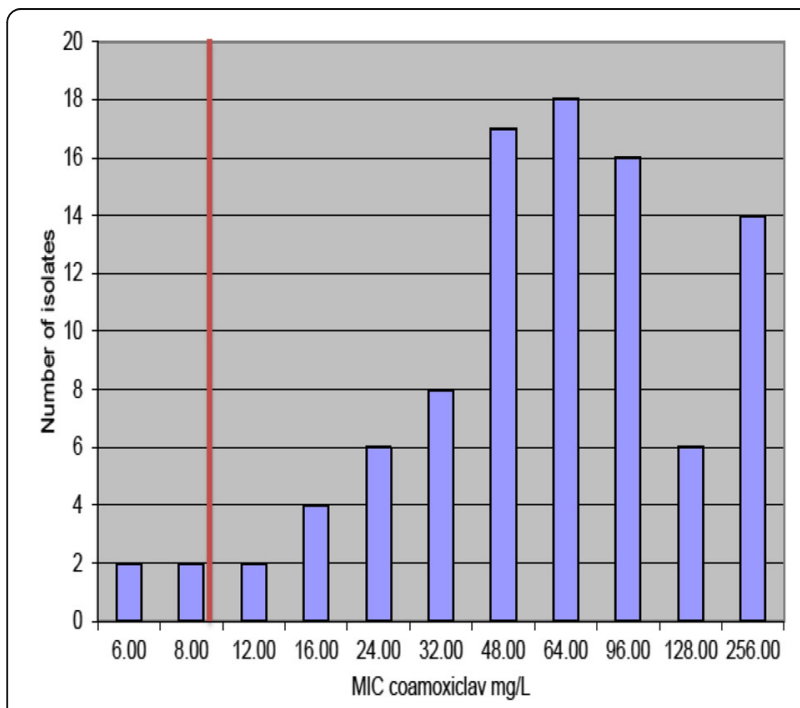

Fig. 2 Distribution of the mean inhibitory concentration (MIC) of 95 Extended spectrum beta-lactamase (ESBL) producing isolates. EUCAST break-point of $8 \mathrm{mg} / \mathrm{L}$ demonstrated by red vertical line
Table 1 Clinico-demographic profile of patients isolated with extended-spectrum beta lactamase producing urinary infections

\begin{tabular}{lll}
\hline Average age of patients (yrs) & $\%$ & $\mathrm{~N}=$ \\
\hline Female & 66.25 & n.a. \\
Male & 74.65 & n.a.
\end{tabular}

Source of Isolation of UTI

Emergency Department 14

General Practice $\quad 36 \quad 34$

Inpatient Wards $26 \quad 25$

$\begin{array}{lll}\text { Nursing Home } & 24 & 23\end{array}$

Species Isolated

$\begin{array}{lll}\text { Klebsiella pneumoniae } & 20 & 19\end{array}$

Escherichia coli

$80 \quad 76$

plate. The E test results showed mecillinam did not inhibit the isolate at even $256 \mathrm{mg} / \mathrm{L}$ but co-amoxiclav inhibited 349 at $128 \mathrm{mg} / \mathrm{L}$ alone.

\section{Discussion}

The increasing prevalence of clinically significant antibiotic resistant bacteria, especially for those resistant to multiple classes of antibiotics, makes appropriate antimicrobial treatment challenging $[25,26]$. Many pathogens resistant to first line agents, then require broader spectrum, more expensive agents with less favorable safety profiles, which in turn accelerates the generation of multi drug resistant (MDR) pathogens, where no agents may be available to treat infections caused by these microorganisms. As the ESBL resistance mechanism is commonly expressed by plasmid encoded B lactamases, resistance can be easily transferred between other bacterial species by horizontal gene transfer. These plasmids frequently carry genes encoding for resistance to other antibiotic classes. Unlike methicillin resistant Staphylococcus aureus, the resistant Gram-negative bacteria commonly colonise the bowel and there is no available decolonization procedure. Long-term colonization by these bacteria ensures these patients remain a potential source of transmission for many years, with potentially indefinite requirement for isolation and contact precautions $[27,28]$.

Table 2 Fractional inhibitory concentration (FIC) of the five extended-spectrum beta lactamase producing isolates which were resistant to mecillinam monotherapy

\begin{tabular}{ll}
\hline Isolate & FIC \\
\hline 842 & Synergy \\
513 & Synergy \\
779 & Indifference \\
349 & Indifference \\
031 & Indifference \\
\hline
\end{tabular}


There is an urgent need for more research into new agents that are effective against ESBLs and other resistant bacteria. With the increase in multidrug and carbapenemresistant microorganisms, there is a need to minimize carbapenem use and they should not be first-line choice for treating ESBLs [29]. Europe-wide antimicrobial drug resistance rates remain high. A Greek study carried out in 2007 demonstrated CRE in $70 \%$ of hospitals. Pan-drug resistant $K$. pneumoniae have also been reported in the same population. The use of mecillinam for UTIs is promising as pivmecillinam achieves high levels in urine, has a low resistance profile and inhibited $94.74 \%$ of the ESBLs in this study. However, a broader clinical evaluation in an Irish patient population is required before clinical use can be advocated. Mecillinam like other penicillins, has low toxicity like other penicillins and is also safe to use for the treatment of UTIs in pregnancy [30]. Mecillinam is poorly absorbed from the gastrointestinal tract but pivmecillinam is well absorbed. Pivmecillinam reaches serum concentrations of 3ug/ml $1.5 \mathrm{~h}$ after $200 \mathrm{mg}$ taken orally [31].

Inappropriate use of broad-spectrum antibiotics such as carbapenems adds to the problem of antimicrobial resistance as well as being responsible for a rise in C. difficile infections and healthcare costs. This study showed that mecillinam alone has good in vitro activity against clinical isolates of ESBL producing E. coli and Klebsiella.

Carbapenems are often used to treat UTIs caused by ESBLs; however this has been shown to lead to further resistance and will exacerbate the CRE problem. Invasive CRE infections have limited therapeutic options with mortality rates in excess of $40 \%$ [32]. Pivmecillinam, widely used in Scandinavian countries for treating uncomplicated UTIs has a good safety profile [33]. Side effects of mecillinam include rash, nausea and vomiting. Surveillance in countries that strictly limit pivmecillinam for acute uncomplicated UTIs has shown low resistance in the community despite widespread use for more than 20 years [16]. There are reports of mecillinam resistance in E. coli; one mechanism is increased levels of ppGpp, a nucleotide effector [34]. Other studies have shown a low probability of future clonal spread of mecillinam resistance due to little association of resistance due to specific clonal groups. Other authors found that mecillinam resistance is not associated with ESBL production [35, 36].

In this study only $5.36 \%$ of ESBLs that were E tested were resistant to mecillinam according to the EUCAST breakpoints with only two isolates having an MIC of $>256 \mathrm{mg} / \mathrm{L}$. This may be due the fact that mecillinam is only slightly affected by TEM and SHV1, which are the most frequent beta lactamases found in E. coli. In a French study, clinical efficiency was high with pivmecillinam and independent of MIC, which suggests susceptibility testing for UTIs caused by E. coli is not required with pivmecillinam, and could possibly be given empirically [17]. The results of the E tests with coamoxiclav show that only $2.11 \%$ of isolates were susceptible to the drug according to the CLSI breakpoints, however according to the BSAC breakpoints, $25 \%$ would be effective treatment of UTIs. Resistance to coamoxiclav is common and in a Spanish study $30.6 \%$ of its ESBLs population were non-susceptible. The principle risk factor in that study was previous use of coamoxiclav, confirming that increased consumption leads to increased resistance. Resistance mechanisms of $E$. coli to coamoxiclav include B-lactamase overproduction, AmpC cephalosporinase hyperproduction and inhibitor-resistant penicillinases [37]. Resistance is acquired by clonal and non-clonal spread, dissemination of mobile elements with different bla genes and eventual mutations in individuals organisms [38].

Coamoxiclav alone was ineffective against $97.89 \%$ of the isolates tested and should not be used alone for treatment of ESBL positive UTIs, however, the combination of coamoxiclav and mecillinam decreased the MIC significantly compared to mecillinam or coamoxiclav alone in three isolates. However two isolates had higher MICs with the combination of the two antibiotics compared to treatment with one antibiotic. For isolate 779 the coamoxiclav MIC increased from $128 \mathrm{mg} / \mathrm{L}$ alone to $>256 \mathrm{ug} / \mathrm{ml}$ when combined with mecillinam. Similarly but more worryingly isolate 031 was inhibited by $12 \mathrm{mg} /$ $\mathrm{L}$ of mecillinam alone in the $\mathrm{E}$ test but was not inhibited by $256 \mathrm{ug} / \mathrm{ml}$ in combination with coamoxiclav. The results for the mecillinam and coamoxiclav only in the 96 well plate differed to the results of the drugs alone in the $\mathrm{E}$ test. It is conceivable that some of these infections could represent identical bacterial strains, however, patients were disparate in time and place, and therefore it is likely that they represent different strains. This demonstrates that the combination of coamoxiclav and pivmecillinam on different species that produce ESBLs could be beneficial. However, further studies using mecillinam monotherapy or in combination with clavulanic acid, would benefit with a larger sample size to improve statistical power.

\section{Conclusion}

Pivmecillinam alone appears to be a suitable treatment for UTIs due to ESBL producing Enterobacteriaceae especially lower UTIs. Resistance to this agent has been low even though it has been used for treating UTIs in Nordic countries for more than 20 years. In the future pivmecillinam in combination with coamoxiclav, would seem to be an appropriate treatment alternative for Irish ESBL infections and will also help reduce carbapenem usage, which in turn should help reduce the generation of CRE producing organisms. However for combination therapy further clinical evaluation would be required before clinical use could be advocated. 


\section{Abbreviations}

CRE: Carbapenem Resistant Enterobacteriaceae; ESBL: Extended spectrum beta lactamase; EUCAST: European Committee on Antimicrobial Susceptibility Testing; FIC: Fractional inhibitory concentration; MIC: Mean inhibitory concentration; PBP: Penicillin binding protein; SHV: Sulfhydryl variable beta-lactamases; TEM: Temoneira beta-lactamases; UTI: Urinary tract infection

\section{Acknowledgements}

We would like to acknowledge the tremendous work of the laboratory microbiologists who identified all the organisms and conducted the original susceptibility testing for all the organisms used in this study.

\section{Funding}

No funding was received at any stage for this study.

\section{Availability of data and materials}

The vast majority of the data supporting our findings are contained within the manuscript. The remaining data will not be shared at present as it lies central to future research and manuscripts. Identifying/confidential patient data would not be available.

\section{Authors' contributions}

All authors have read and approved the manuscript. FOK, SK and JF made substantial contributions to conception and design, or acquisition of data, or analysis and interpretation of data. RPM and JAT were involved in drafting the manuscript or revising it critically for important intellectual content. JAT and JF gave final approval of the version to be published.

\section{Competing interests}

The authors declare that they have no competing interests.

\section{Consent for publication}

Not applicable.

\section{Ethics approval and consent to participate}

This study involving the use of patient-derived samples is in line with the Helsinki Declaration. Ethical approval was granted by the Tallaght University Hospital Ethics Committee. Written consent was obtained at the outset and participants could withdraw from the study at any time.

\section{Author details}

${ }^{1}$ Department of Clinical Microbiology, AMNCH, Tallaght Hospital, Dublin 24, Ireland. ${ }^{2}$ Department of Urological Surgery, AMNCH, Tallaght Hospital, Dublin 24 , Ireland.

\section{Received: 4 September 2015 Accepted: 23 August 2016}

\section{Published online: 03 November 2016}

\section{References}

1. Dhillon RH-P, Clark J. ESBLs: a clear and present danger? Crit Care Res Pract. 2012;2012:625170

2. Livermore DM, Hawkey PM. CTX-M: changing the face of ESBLs in the UK J Antimicrob Chemother. 2005;56:451-4.

3. Fennell J, et al. Increasing prevalence of ESBL production among Irish clinical Enterobacteriaceae from 2004 to 2008: an observational study. BMC Infect Dis. 2012:12:116

4. Linhares I, Raposo T, Rodrigues A, Almeida A. Frequency and antimicrobial resistance patterns of bacteria implicated in community urinary tract infections: a ten-year surveillance study (2000-2009). BMC Infect Dis. 2013;13:19.

5. Thomson KS. Extended-spectrum-beta-lactamase, AmpC, and Carbapenemase issues. J Clin Microbiol. 2010:48:1019-25.

6. Kanj SS, Kanafani ZA. Current concepts in antimicrobial therapy against resistant gram-negative organisms: extended-spectrum beta-lactamaseproducing Enterobacteriaceae, carbapenem-resistant Enterobacteriaceae, and multidrug-resistant Pseudomonas aeruginosa. Mayo Clin Proc 2011;86:250-9.

7. Pobiega $\mathrm{M}$, et al. Molecular characterization and drug resistance of Escherichia coli strains isolated from urine from long-term care facility residents in Cracow, Poland. Med Sci Monit. 2013;19:317-26.
8. Martinez P, Garzón D, Mattar S. CTX-M-producing Escherichia coli and Klebsiella pneumoniae isolated from community-acquired urinary tract infections in Valledupar, Colombia. Braz J Infect Dis. 2012;16:420-5.

9. Azap OK, et al. Risk factors for extended-spectrum beta-lactamase positivity in uropathogenic Escherichia coli isolated from community-acquired urinary tract infections. Clin Microbiol Infect. 2010;16:147-51.

10. Pelly $\mathrm{H}$, et al. Outbreak of extended spectrum beta-lactamase producing $\mathrm{E}$. coli in a nursing home in Ireland, May 2006. Euro Surveill. 2006:11:E060831.1.

11. Ludden C, et al. Colonisation with ESBL-producing and carbapenemaseproducing Enterobacteriaceae, vancomycin-resistant enterococci, and meticillin-resistant Staphylococcus aureus in a long-term care facility over one year. BMC Infect Dis. 2015;15:168.

12. EARS-Net Report, Quarter 1-4 2015. Health Protection Surveillance Centre 2016 http://www.hpsc.ie/A-Z/MicrobiologyAntimicrobialResistance/European AntimicrobialResistanceSurveillanceSystemEARSS/EARSSSurveillanceReports/ 2015Reports/File,15214,en. Accessed June 2016

13. Al-Assil B, Mahfoud M, Hamzeh AR. Resistance trends and risk factors of extended spectrum $\beta$-lactamases in Escherichia coli infections in Aleppo, Syria. Am J Infect Control. 2013;41:597-600.

14. Hoşbul T, et al. In vitro activity of fosfomycin trometamol in the treatment of Escherichia coli related uncomplicated urinary tract infections. Mikrobiyol Bul. 2009;43:645-9.

15. Pallett A, Hand K. Complicated urinary tract infections: practical solutions for the treatment of multiresistant Gram-negative bacteria. J Antimicrob Chemother. 2010;65(Suppl 3):iii25-33.

16. Auer S, Wojna A, Hell M. Oral treatment options for ambulatory patients with urinary tract infections caused by extended-spectrum-betalactamase-producing Escherichia coli. Antimicrob Agents Chemother. 2010;54:4006-8

17. Thomas K, Weinbren MJ, Warner M, Woodford N, Livermore D. Activity of mecillinam against ESBL producers in vitro. J Antimicrob Chemother. 2006; 57:367-8

18. Graninger W. Pivmecillinam-therapy of choice for lower urinary tract infection. Int J Antimicrob Agents. 2003:22(Suppl 2):73-8.

19. Papp-Wallace KM, Endimiani A, Taracila MA, Bonomo RA. Carbapenems: past, present, and future. Antimicrob Agents Chemother. 2011:55:4943-60.

20. Meletiadis J, Pournaras S, Roilides E, Walsh TJ. Defining fractional inhibitory concentration index cutoffs for additive interactions based on self-drug additive combinations, Monte Carlo simulation analysis, and in vitro-in vivo correlation data for antifungal drug combinations against Aspergillus fumi. Antimicrob Agents Chemother. 2010;54:602-9.

21. Hall MJ, Middleton RF, Westmacott D. The fractional inhibitory concentration (FIC) index as a measure of synergy. J Antimicrob Chemother. 1983;11:427-33.

22. White RL, Burgess DS, Manduru M, Bosso JA. Comparison of three different in vitro methods of detecting synergy: time-kill, checkerboard, and E test. Antimicrob Agents Chemother. 1996:40:1914-8.

23. Prieto J, et al. In vitro activities of co-amoxiclav at concentrations achieved in human serum against the resistant subpopulation of heteroresistant Staphylococcus aureus: a controlled study with vancomycin. Antimicrob Agents Chemother. 1998:42:1574-7.

24. Neuzillet $Y$, Naber KG, Schito G, Gualco L, Botto H. French results of the ARESC study: clinical aspects and epidemiology of antimicrobial resistance in female patients with cystitis. Implications for empiric therapy. Med Mal Infect. 2012:42:66-75.

25. Cullen IM, et al. The changing pattern of antimicrobial resistance within 42,033 Escherichia coli isolates from nosocomial, community and urology patient-specific urinary tract infections, Dublin, 1999-2009. BJU Int. 2012;109: 1198-206.

26. Frakking FNJ, et al. Appropriateness of empirical treatment and outcome in bacteremia caused by extended-spectrum- $\beta$-lactamase-producing bacteria. Antimicrob Agents Chemother. 2013;57:3092-9.

27. Fan $\mathrm{N}-\mathrm{C}$, et al. Rise of community-onset urinary tract infection caused by extended-spectrum $\beta$-lactamase-producing Escherichia coli in children. J Microbiol Immunol Infect. 2013. doi:10.1016/j.jmii.2013.05.006.

28. Yamamoto A. Extended-spectrum $\beta$-lactamase (ESBL)-producing Escherichia coli is frequently detected as a pathogen of urinary tract infection in nursing home residents. Nihon Ronen Igakkai Zasshi. 2011:48:530-8.

29. Dewar S, Reed LC, Koerner RJ. Emerging clinical role of pivmecillinam in the treatment of urinary tract infection in the context of multidrug-resistant bacteria. J Antimicrob Chemother. 2014;69:303-8. 
30. Heikkilä A, Pyykkö K, Erkkola R, lisalo E. The pharmacokinetics of mecillinam and pivmecillinam in pregnant and non-pregnant women. $\mathrm{Br} J$ Clin Pharmacol. 1992;33:629-33.

31. Andrews J, Kendall MJ, Mitchard M. Factors influencing the absorption and disposition of mecillinam and pivmecillinam in man. Br J Clin Pharmacol. 1976;3:627-32.

32. Kumarasamy KK, et al. Emergence of a new antibiotic resistance mechanism in India, Pakistan, and the UK: a molecular, biological, and epidemiological study. Lancet Infect Dis. 2010;10:597-602.

33. Lampri $\mathrm{N}$, et al. Mecillinam/clavulanate combination: a possible option for the treatment of community-acquired uncomplicated urinary tract infections caused by extended-spectrum $\beta$-lactamase-producing Escherichia coli. J Antimicrob Chemother. 2012;67:2424-8.

34. Poulsen HO, Johansson A, Granholm S, Kahlmeter G, Sundqvist M. High genetic diversity of nitrofurantoin- or mecillinam-resistant Escherichia coli indicates low propensity for clonal spread. J Antimicrob Chemother. 2013; 68:1974-7.

35. Sougakoff $W$, Jarlier $V$. Comparative potency of mecillinam and other beta-lactam antibiotics against Escherichia coli strains producing different beta-lactamases. J Antimicrob Chemother. 2000;46(Suppl 1):9-14. discussion 63-5.

36. Kariuki $\mathrm{S}$, et al. Escherichia coli from community-acquired urinary tract infections resistant to fluoroquinolones and extended-spectrum betalactams. J Infect Dev Ctries. 2007;1:257-62.

37. Rodríguez-Baño J, et al. Epidemiological and clinical complexity of amoxicillin-clavulanate-resistant Escherichia coli. J Clin Microbiol. 2013; 51:2414-7.

38. Miyakis S, Pefanis A, Tsakris A. The challenges of antimicrobial drug resistance in Greece. Clin Infect Dis. 2011;53:177-84.

\section{Submit your next manuscript to BioMed Central and we will help you at every step:}

- We accept pre-submission inquiries

- Our selector tool helps you to find the most relevant journal

- We provide round the clock customer support

- Convenient online submission

- Thorough peer review

- Inclusion in PubMed and all major indexing services

- Maximum visibility for your research

Submit your manuscript at www.biomedcentral.com/submit 